\title{
Deutschland in der EU: Europas unverzichtbarer Hegemon?
}

\author{
Simon Bulmer
}

Germany has been at the heart of European integration since Chancellor Adenauer's commitment to "Westbindung" in the early 1950s. In the 2010s it has become the European Union's (EU) indispensable power. Indispensability has placed responsibilities upon the federal government but has also brought increased controversy in relation to Germany's role in the EU: both internationally and domestically. This contribution seeks to take stock of Germany's role in the EU during an era of increased European and global uncertainty. Is it credible to regard Germany as the EU's hegemon in the 2010s? Further, what are the effects of the more politicised nature of European policy in domestic politics on Germany playing such a role?

In den 2010er Jahren rückte die Rolle Deutschlands in der Europäischen Union (EU) zunehmend in den Mittelpunkt. Auf der einen Seite stand die Sorge, die der damalige polnische Außenminister Radosław Sikorski im Jahr 2011 während der Krise in der Eurozone äußerte: „I fear Germany's power less than her [sic!] inactivity. “1 Auf der anderen Seite wurde in Griechenland während der Eurozonenkrise die deutsche Führungsrolle verteufelt. ${ }^{2}$ Italienische Autoren veröffentlichten sogar ein Buch, in dem auf die Entstehung eines „Vierten Reiches“ angespielt wird. ${ }^{3}$ Ob positiv oder kritisch betrachtet - Deutschland gilt als unverzichtbare Gestaltungsmacht der EU. Damit verbunden sind gestiegene Erwartungen, aber auch Bedenken. Angesichts der noch lange nicht überwundenen Krisen in der EU und der langen Kanzlerschaft Angela Merkels, die sich langsam dem Ende neigt, erscheint es angebracht, die Grundlagen der deutschen Rolle in der EU darzustellen.

Von entscheidender Bedeutung ist dabei die Frage, von welchem Verständnis der deutschen Führungsmacht in der EU auszugehen ist. Diese Macht manifestiert sich auf unterschiedliche Weise: durch Diplomatie, als strukturelle Macht und durch die Kraft der Ideen. Sie unterliegt aber auch einer Reihe von Einschränkungen, die sich sowohl aus der Struktur der EU als auch aus der deutschen Politik ergeben. All diese Faktoren wirken sich auf die Fähigkeit Deutschlands aus, zur Suche nach Lösungen für die „Polykrise“ der EU beizutragen. ${ }^{4}$

Im ersten Abschnitt wird der veränderte historische Kontext der Rolle Deutschlands in Europa betrachtet, um dann die verschiedenen, für die EU-Mitgliedschaft relevanten As-

* Prof. Dr. Simon Bulmer, Department of Politics, University of Sheffield.

Dieser Beitrag basiert auf dem kürzlich erschienenen Buch von Simon Bulmer und William Paterson (Simon Bulmer/William Paterson: Germany and the European Union: Europe's Reluctant Hegemon?, London 2019) und stellt deren gemeinsame Arbeit vor. Der Autor des Beitrags würdigt die langjährige produktive Zusammenarbeit mit Willie, wenngleich dieser für mögliche Fehler oder Auslassungen in diesem Artikel nicht verantwortlich ist.

1 Radosław Sikorski: I fear Germany's power less than her inactivity, in: Financial Times, 28. November 2011.

2 Vgl. Claudia Sternberg/Kira Gartzou-Katsouyanni/Kalypso Nicolaidis: The Greco-German Affair in the Euro Crisis. Mutual Recognition Lost?, London 2018, S. 42ff.

3 Vittorio Feltri/Gennaro Sangiuliano: Il Quarto Reich. Come la Germania ha sottomesso l'Europa, Mailand 2014. Auf Deutsch: „Das Vierte Reich: Wie Deutschland Europa unterworfen hat“.

4 Europäische Kommission: Speech by President Jean-Claude Juncker at the Annual General Meeting of the Hellenic Federation of Enterprises, 21. Juni 2016, abrufbar unter: http://europa.eu/rapid/pressrelease_SPEECH-16-2293_en.htm (letzter Zugriff: 19.5.2017). 
pekte von Macht zu untersuchen. Danach werden die Grenzen der Gestaltungsmacht und der Rolle Deutschlands, die sich aus dem EU-System selbst sowie aus der Innenpolitik ergeben, betrachtet. Der Beitrag wendet sich sodann dem Verhalten Deutschlands auf europäischer Ebene zu und konzentriert sich hierbei vor allem auf die Krisen in der EU in den vergangenen Jahren. Um einen Eindruck vom Charakter der deutschen Gestaltungsmacht zu erhalten, wird analysiert, wie sich das Verhältnis zwischen deutscher Führungsrolle und innenpolitischen Zwängen entwickelt hat. Konkret stellt der Artikel folgende Fragen: Kann Deutschland als Hegemon Europas betrachtet werden? Erleichtert oder verhindert die deutsche Innenpolitik diese Rolle?

\section{Der historische Kontext}

Die deutsche Machtfrage ist zentraler Bestandteil des europäischen Integrationsprozesses. Schon zu Beginn war sie ein besonderes Anliegen der anderen Staaten, vor allem Frankreichs. Die eigentliche Attraktivität der im Schuman-Plan dargelegten supranationalen Methode bestand darin, die Schwerindustrien der Mitgliedstaaten (im Bereich Kohle und Stahl) gemeinsam zu steuern, den gegenseitigen Zugang zur Rohstoffversorgung zu ermöglichen und eine neue deutsch-französische Partnerschaft als Kern der Integration aufzubauen. Die supranationale Methode etablierte einen Integrationskurs, der den wirtschaftlichen und politischen Interessen Westdeutschlands diente. In Verbindung mit den Realitäten des Kalten Krieges in einem geteilten Europa beruhigte die supranationale Einbeziehung der jungen Bundesrepublik die Sorgen der Nachbarn angesichts der jüngsten Vergangenheit.

Die europäische Integration war ein wichtiges Instrument, um die deutsche Gestaltungsmacht unter Kontrolle zu halten. ${ }^{5}$ Vor allem bis zur Wiedervereinigung versuchte die Bundesrepublik Deutschland lange Zeit, sich durch den „leadership avoidance reflex" selbst einzuschränken. ${ }^{6}$ Die europäische Integration wurde zur Aufwärtsspirale: Sie beruhigte die Sorgen der westlichen Nachbarn in Bezug auf die deutsche Führungsrolle und bot zugleich dem neuen westdeutschen Staat einen vielversprechenden multilateralen Rahmen, um seine politischen Ziele zu verfolgen. Die wirtschaftliche Integration erwies sich durch die Schaffung eines großen Binnenmarkts, welcher die Entwicklung Westdeutschlands als exportorientierte Wirtschaft (als „extraordinary trader“) unterstützte, als komplementär. ${ }^{7}$ Politisch half die europäische Integration Deutschland dabei, dem Schatten der Vergangenheit zu entkommen, und den außenpolitischen Beziehungen diente sie als „Emanzipationsvehikel“" ${ }^{8}$ Es ist daher nicht verwunderlich, dass die aufeinanderfolgenden Bundesregierungen lange Zeit innenpolitische Unterstützung für die europäische Integration fanden, da

5 Vgl. Hans-Peter Schwarz: Die gezähmten Deutschen: von der Machtbesessenheit zur Machtvergessenheit, Stuttgart 1985; Peter Katzenstein (Hrsg.): Tamed Power: Germany in Europe, Ithaca 1997.

6 William Paterson: Muß Europa Angst vor Deutschland haben?, in: Rudolf Hrbek (Hrsg.): Der Vertrag von Maastricht in der wissenschaftlichen Kontroverse. Beiträge für das Jahreskolloquium des Arbeitskreises Europäische Integration e.V., 12.-14. November 1992 in Bonn, Baden-Baden 1993, S. $9 \mathrm{ff}$.

7 Wolfgang Hager: Germany as an Extraordinary Trader, in: Wilfrid Kohl/Giorgio Basevi (Hrsg.): West Germany, a European and global power, Lexington 1980, S. 3ff.

8 Elfriede Regelsberger/Wolfgang Wessels: Die Europäische Politische Zusammenarbeit (EPZ) - Emanzipationsvehikel, Koalition oder Integrationsrahmen für die Außenpolitik Bonns, in: Rudolf Hrbek/Wolfgang Wessels (Hrsg.): EG-Mitgliedschaft: ein vitales Interesse der Bundesrepublik Deutschland?, Bonn 1984, S. 389ff., hier S. $391 \mathrm{ff}$. 
diese eindeutige politische und wirtschaftliche Vorteile mit sich brachte. Dies ging sogar so weit, dass Europa zu einer „surrogate identity, replacing German nationalism“ wurde. ${ }^{9}$

Das „Krisenjahrzehnt“ - die 2010er Jahre - hat einige der grundlegenden Annahmen über die deutsche Europapolitik in Frage gestellt. Natürlich gehen einige der Veränderungen auf die Zeit vor 2010 zurück. Am weitreichendsten war der Wegfall der von außen auferlegten Einschränkungen der deutschen Souveränität mit dem Ende des Kalten Krieges und der deutschen Wiedervereinigung. Dennoch änderte sich Deutschlands europapolitische Rolle nicht plötzlich, da Bundeskanzler Helmut Kohls Bekenntnis zu einer Vertiefung der Integration die Kontinuität der bereits bestehenden Integrationsstrategie betonte. Die deutsche Politik der Zurückhaltung wurde erst während der Kanzlerschaft Gerhard Schröders überdacht. Schröder wies darauf hin, dass Deutschland großes Interesse daran habe, sich als bedeutende Führungsnation in Europa hervorzutun und seine Außenpolitik entsprechend zu gestalten. ${ }^{10}$ Zumindest auf rhetorischer Ebene erlebte die Schröder-Ära im Rahmen der „Normalisierung“ der deutschen Außen- und Europapolitik daher eine stärkere Betonung nationaler Interessen. ${ }^{11} \mathrm{Zu}$ diesem Zeitpunkt ließen Verweise auf die „Vereinigten Staaten von Europa“ oder auf ein föderalistisches Konzept der EU nach. Die Rede von Außenminister Joschka Fischer an der Humboldt-Universität zu Berlin aus dem Jahr 2000 ist eine der letzten, die solche Elemente enthält. ${ }^{12}$

Nach der langen Verfassungsdebatte und dem Inkrafttreten des Vertrags von Lissabon stellte besonders das politische Umfeld der EU die deutsche Europapolitik vor Herausforderungen. Die Krise in der Eurozone, die Ukraine-Krise, die Flüchtlingskrise, Bedenken hinsichtlich der Rechtsstaatlichkeit in Ungarn und insbesondere in Polen, das Referendum der britischen WählerInnen im Juni 2016 über den Austritt aus der EU und der Aufstieg populistischer Parteien forderten die Politiken und Werte heraus, die im Mittelpunkt der Bonner bzw. Berliner Politik stehen. Auf der internationalen Bühne haben die Schwierigkeiten, welche die Präsidentschaft Donald Trumps für die globale Wirtschaftsordnung und das westliche Bündnis darstellt, auch erhebliche Auswirkungen auf die EU und Deutschland. Diesen Herausforderungen sieht sich nun eine deutsche Koalitionsregierung gegenüber, die aus der Wahl im September 2017 deutlich geschwächt hervorging. Beide große Volksparteien verzeichneten starke Verluste und eine zunehmende Politisierung der Europapolitik wurde deutlich. Dies wirkt sich auf Deutschlands Rolle in der EU aus und in Anbetracht der Bedeutung Deutschlands für die europäische Integration auch auf die Entwicklung der EU.

9 Andrei Markovits/Simon Reich: The German Predicament: Memory and Power in the New Europe, Ithaca 1997, S. 39.

10 Vgl. Roger Cohen: A New German Assertiveness On Its Foreign Policy Stance, in: The New York Times, 12. September 1999; Katy Crossley-Frolick: Revisiting and Reimagining the Notion of Responsibility in German Foreign Policy, in: International Studies Perspectives 4/2017, S. 443ff., hier S. 457.

11 Adrian Hyde-Price/Charlie Jeffery: Germany in the European Union: Constructing Normality, in: Journal of Common Market Studies 4/2001, S.689ff.; Simon Bulmer/William Paterson: Germany and the European Union: from 'tamed power' to normalized power?, in: International Affairs 5/2010, S. $1051 \mathrm{ff}$.

12 Joschka Fischer: "From Confederacy to Federation - Thoughts on the finality of European integration". Speech by Joschka Fischer at the Humboldt University in Berlin, 12 May 2000, abrufbar unter: http://ec.europa.eu/ dorie/fileDownload.do;jsessionid=9TXVSzSHrJ4WmfrgWXjLpLNZSnXQtGypBkh2S2GGnD3K9MW6wybp! 1615003456?docId=192161\&cardId=192161 (letzter Zugriff: 18.1.2019). 
Im vorliegenden Artikel wird argumentiert, dass Deutschland über beträchtliche Machtressourcen und strukturelle Macht in Europa verfügt. ${ }^{13}$ Die Rolle der Bundesregierung auf europäischer Ebene erfährt jedoch Einschränkungen, da sie von den Partnern legitimiert werden muss. Zudem stellt auch die zunehmende innerstaatliche Politisierung der EU-Politik ein Hindernis dar. Die Rolle Deutschlands als unverzichtbarer Hegemon lässt sich am deutlichsten an bestimmten Phasen der Krise in der Eurozone festmachen. Für eine Gesamtbewertung eignet sich dieser Befund allerdings nur begrenzt.

\section{Aspekte von Macht}

Die Rolle Deutschlands in der EU ist stark geprägt von dessen Auftreten als führender Mitgliedstaat während der Krise in der Eurozone. Die relative Konjunkturschwäche Frankreichs war damals offensichtlich und Deutschland nahm bei der Gestaltung der neuen Fiskalregeln eine bedeutende Rolle ein. Nach seinem Amtsantritt im Jahr 2017 versuchte Präsident Emmanuel Macron zwar, mit neuer Dynamik die Agenda der EU zu gestalten, dies stieß jedoch auf zwei Probleme: die mangelnde deutsche Unterstützung seiner Vorschläge zur Governance der Eurozone sowie das spätere Aufkommen der Proteste der „Gelbwesten“" in Frankreich, die die Autorität des Präsidenten geschwächt haben. Diese Situation bedeutet jedoch nicht automatisch, dass nun Deutschland die Führungsrolle in der EU einfach so übernehmen kann. Denn es ist eine Sache, der mächtigste Staat in der EU zu sein. Diese Führungsrolle dann auch einzunehmen, ist etwas anderes. Um dies zu verdeutlichen, werden die verschiedenen Dimensionen der Macht der Mitgliedstaaten in der EU untersucht.

Dazu ist es zunächst notwendig, „Macht“ zu definieren, da diese unterschiedlich verstanden werden kann. Hier wird auf das Konzept von Macht als Hegemonie zurückgegriffen. Dieses Konzept umfasst das Führen und das Herrschen, wobei es fließende Übergänge gibt. ${ }^{14}$ Im vorliegenden Artikel werden vier verschiedene Aspekte von Hegemonie herangezogen, um die deutsche Führungsrolle in der EU zu untersuchen. Der erste leitet sich aus der Theorie der hegemonialen Stabilität ab. Diese greift auf Analysen der Rolle zurück, welche die Vereinigten Staaten bei der Übernahme von Führungsaufgaben zur Stabilisierung der Weltwirtschaft im zwanzigsten Jahrhundert gespielt haben. ${ }^{15}$ Übertragen auf den EU-Kontext würde dies bedeuten, dass Deutschland eine führende Rolle beim Versuch der Stabilisierung der EU spielt, indem es vielleicht die diplomatische Rolle übernimmt, welche die deutsch-französische Partnerschaft in früheren Zeiten der europäischen Integration gespielt hat. ${ }^{16}$ Um diese Rolle einzunehmen, muss der führende Staat über ausreichende wirtschaftliche Ressourcen verfügen und bereit sein, diese in die Bereitstellung von „Kollektivgütern“ zu investieren, um die Stabilität des EU-Systems zu gewährleisten. Der aktive Ein-

13 Indem sie sich auf die Konzepte von Macht und Hegemonie konzentriert, hat die Analyse einen weiteren Geltungsbereich als mit einem Fokus auf das Konzept der politischen Führung.

14 Siehe Perry Anderson: The H-Word: The Peripeteia of Hegemony, London 2017.

15 Siehe z.B. Charles Kindleberger: The World in Depression, 1929-1939, Berkeley/Los Angeles 1973; Robert O. Keohane: The Theory of Hegemonic Stability and Changes in International Economic Regimes, 1967-77, in: Ole Holsti/Randolph Siverson/Alexander George (Hrsg.): Change in the International System, Boulder 1980, S. $131 \mathrm{ff}$.

16 Vgl. Ulrich Krotz/Joachim Schild: Shaping Europe: France, Germany, and Embedded Bilateralism from the Elysée Treaty to Twenty-First Century Politics, Oxford 2013; Thomas Pedersen: Germany, France and the Integration of Europe: A Realist Interpretation, London 1998. 
satz von Diplomatie und der zugrunde liegenden wirtschaftlichen Ressourcen ist daher für eine solche Rolle erforderlich.

Eine weitere potenzielle Form der Führung ergibt sich nach Antonio Gramsci, bei der eine Gruppe (oder eine Gesellschaftsschicht) intellektuelle und moralische Führung bietet. ${ }^{17}$ Diese Form der Führung betont Ideologie und Wertesysteme. Im EU-Kontext würde sie den Schwerpunkt auf eine bestimmte Vision der EU im Allgemeinen legen, so zum Beispiel auf Fischers Humboldt-Rede. Ebenso könnte es sich z.B. um die ordoliberal inspirierten Ideen des „sound money“ in Bezug auf die Eurozone handeln.

Würde Deutschland die Rolle des Hegemonen spielen, sei es durch diplomatische Initiativen oder durch Ideen, würde dies die Zustimmung oder zumindest die Akzeptanz der Partner erfordern. Denn ohne die nötige Legitimität ist hegemoniale Stabilität nicht gewährleistet. ${ }^{18}$ Dieser Vorschlag bringt zwei besondere Aspekte mit sich, die im Zusammenhang mit der EU untersucht werden müssen: die systemische und die politische Legitimität. Kurz gesagt betrifft die systemische Legitimität die Frage, inwieweit die EU als System konzipiert und in der Lage ist, einen Staat hinzunehmen, der hegemoniale Stabilität bietet. Im Gegensatz dazu geht es bei der politischen Legitimität darum, ob andere Mitgliedstaaten oder ihre BürgerInnen bereit sind, diese hegemoniale Rolle zu akzeptieren.

Ein letzter Aspekt bezüglich der Macht von Mitgliedstaaten ist die Innenpolitik. Die Ursprünge des Konzepts der Hegemonie reichen bis zu den Stadtstaaten im antiken Griechenland zurück. Schon zu dieser Zeit wurde festgestellt, dass ein Stadtstaat die Rolle des Hegemonen nicht spielen kann, wenn ihm die innere Stabilität fehlt. So wird beispielsweise der Verlust der Hegemonie Athens an Sparta während des Peloponnesischen Krieges auf „its internal politics and the ensuing deterioration in leadership“ zurückgeführt. ${ }^{19}$ Die Gegebenheiten in der EU sind völlig anders, aber die Grundidee wird klar: Um die führende Rolle einnehmen zu können, ist ein nationaler Konsens bezüglich der Ziele erforderlich. Es ist jedoch schwieriger geworden, diesen Konsens zu erreichen, da die Europapolitik seit Maastricht stärker politisiert ist. Diese Beobachtung unterstreicht einen wichtigen Aspekt, der zum Verständnis der Rolle Deutschlands in der EU beiträgt: Sowohl die Beziehungen Deutschlands zu den Partnern als auch die deutsche Innenpolitik sind relevant. Die folgenden Abschnitte beschäftigen sich daher mit den internationalen, EU-internen und innenpolitischen Aspekten der deutschen Gestaltungsmacht in der EU.

\section{Deutschlands Rolle in einem sich verändernden EU-Umfeld}

Um der Frage nachzugehen, ob Deutschland tatsächlich über die Möglichkeiten verfügt, die Rolle des Hegemonen der EU zu übernehmen, muss zunächst der institutionelle Rahmen der EU berücksichtigt werden. Außerdem muss geprüft werden, ob Deutschland über die notwendigen Ressourcen verfügt, die Voraussetzung für die Wahrnehmung dieser Rolle sind.

17 Howard Lentner: Hegemony and Autonomy, in: Political Studies 4/2005, S. 735ff., hier S. 740.

18 Ian Clark: Hegemony in International Society, Oxford 2011, S. 32.

19 Lentner: Hegemony and Autonomy, 2005, S. $739 f$. 


\section{Der institutionelle Rahmen}

Obwohl die supranationale Integration darauf ausgerichtet war zu verhindern, dass ein Staat eine dominante Rolle spielt, gelang dies nicht immer. Am offensichtlichsten zeigte sich dies an der einflussreichen Rolle des französischen Staatspräsidenten Charles de Gaulle in den 1960er Jahren, der die Entwicklung der EU sowohl in Bezug auf die Vertiefung als auch Erweiterung verhinderte. Mit zunehmender Anzahl an Mitgliedstaaten ist es für einen einzelnen Staat schwieriger geworden, eine solche dominante Rolle zu spielen. Zudem haben sich andere Optionen aufgetan, die eigenen Interessen durchzusetzen. So führte beispielsweise der Widerstand des Vereinigten Königreichs gegen eine Reihe von Politiken, insbesondere gegen den Beitritt zur Währungsunion oder gegen die Abschaffung von Passkontrollen, zu einer Einigung über Opt-outs, die es den willigen Staaten ermöglicht, die Integration zu vertiefen und voranzubringen, während andere nicht teilnehmen müssen. Differenzierte Integration, wie z.B. in der Eurozone, oder „venue shopping“, wie z.B. die Vertiefung der Integration zunächst in einem anderen Format (z.B. beim Schengener Durchführungsübereinkommen oder dem Vertrag von Prüm), sind Optionen, die deutsche Regierungen in der Vergangenheit verfolgt haben. Damit wollten sie vermeiden, dass die Integration durch das „slowest ship in the convoy“ diktiert wird.

Im Einklang mit dem liberalen Intergouvernementalismus besteht für große Mitgliedstaaten die Möglichkeit, ihr politisches Gewicht und ihren Einfluss bei wichtigen EU-Beschlüssen einzusetzen, wie beispielsweise im Europäischen Rat. ${ }^{20}$ Große Staaten haben dieser Logik nach größere Verhandlungsmacht. Sie können eine Einigung blockieren, alternative Politikfelder oder Avantgarde-Gruppen nutzen sowie ihre Machtressourcen einsetzen. Durch Kompensationsleistungen können sie eine Einigung näher an ihren Vorstellungen erreichen. In den 2010er Jahren verfügte Deutschland außerdem mit Kanzlerin Merkel über die herausragende politische Figur in der EU und hatte mit ihr die zentrale Akteurin im Europäischen Rat. Ihre lange Amtszeit war ein besonderer Vorteil für die deutsche Diplomatie, obwohl die Kontroversen um ihre Politik während der Flüchtlingskrise im Jahr 2015 ihre Position schwächten. Das Ergebnis der Bundestagswahlen 2017 hat zudem ihre Macht innenpolitisch untergraben.

\section{Grundlagen deutscher Macht}

Deutschland besitzt die notwendigen ökonomischen Ressourcen, um die Rolle eines „hegemonialen Stabilisators“ zu übernehmen. Als bevölkerungsmäßig größter Mitgliedstaat und mit einem deutlich höheren Bruttoinlandsprodukt (BIP) als andere große Mitgliedstaaten, sowohl insgesamt als auch pro Kopf, verfügt es über eine starke volkswirtschaftliche Basis. Die Arbeitslosigkeit in den 2010er Jahren ist gering. Der Leistungsbilanzüberschuss Deutschlands erreichte bis 20169 Prozent des BIP. Wenn überhaupt, dann ist der Handelsüberschuss des Landes potenziell destabilisierend für die Handelsbeziehungen der EU. ${ }^{21}$ Daten des „Observatory of Economic Complexity“ zeigen, dass Deutschland für 18 der EU-Mitgliedstaaten die wichtigste Importquelle ist. Der gesamtstaatliche Haushalts-

20 Vgl. Andrew Moravcsik: Preferences and Power in the European Community: A Liberal Intergovernmentalist Approach, in: Journal of Common Market Studies 4/1993, S. 473ff., hier S. 496ff.

21 Wade Jacoby: Surplus Germany, Transatlantic Academy: Paper Series 8/2017; Simon Tilford: German rebalancing: Waiting for Godot?, Centre for European Reform: Policy Brief März 2015. 
saldo Deutschlands (in Prozent des BIP) war in den 2010er Jahren stärker als in allen anderen großen Mitgliedstaaten, obwohl sein Defizit 2010 aufgrund von Maßnahmen zur Bewältigung der globalen Finanzkrise 4,2 Prozent erreichte. ${ }^{22}$

Die oben genannten Daten schreiben der deutschen Wirtschaft eine beträchtliche strukturelle Macht zu. Grundlage dafür ist die Integration deutscher Unternehmen in die europäischen Lieferketten. Besonders aufschlussreich ist die Beziehung $\mathrm{zu}$ den vier Visegrád-Staaten (V4). Laut Popławski sind die V4 „Germany’s most important global partner both in exports and imports“. ${ }^{23}$ So sorgt die Auslagerung der Produktion in die Visegrád-Staaten für niedrigere Lohnkosten und deckt den Mangel an FachingenieurInnen zu Hause. Die deutsche verarbeitende Industrie verfügt damit über eine beträchtliche strukturelle Kraft zur Unterstützung der Diplomatie des Landes.

Die Verteidigung ist der einzige Bereich, in dem die deutschen Ressourcen schwächer sind. Mit dem Ende des Kalten Krieges nahm Deutschland eine große „Friedensdividende“ an und seine Verteidigungsausgaben sanken von 2,7 Prozent des BIP (1989) auf 1,2 Prozent (2016). ${ }^{24}$ Trotz des Drucks auf die Bundesregierung, das 2-Prozent-Ziel der NATO zu erreichen, sind nur langsam Fortschritte erkennbar. Dies schwächt die Position Deutschlands hinsichtlich der Einleitung diplomatischer Initiativen in der EU, die eine militärische Dimension haben.

Ideen und Leitbilder sind weitere potenzielle Faktoren für den deutschen Einfluss in der EU. Viele Jahre lang war die deutsche Unterstützung der europäischen Integration an sich ein wichtiges Merkmal. Europäische Lösungen für innenpolitische Probleme wurden aktiv vorangetrieben, wobei die deutsch-französische Partnerschaft als bevorzugtes Instrument diente. ${ }^{25}$ In einigen Fällen - aber nicht allen - hat diese anfängliche Gestaltungsmacht auf das EU-System eingewirkt, was sich nachträglich als Vorteil für Deutschland erwies. Eine ganze Reihe von EU-Politiken entwickelte sich mit starker deutscher Prägung: die Umweltpolitik, das Europäische Währungssystem, die einheitliche Währung, die Justiz- und Innenpolitik einschließlich des Schengen-Raums ohne Passkontrollen und die ursprüngliche Asyl- und Flüchtlingspolitik der EU, durch die eine Änderung des liberalen Artikels 16 des Grundgesetzes (GG) ermöglicht wurde ${ }^{26}$ sowie die Außen- und Sicherheitspolitik.

Bei den Verhandlungen über den Vertrag von Maastricht waren Bundesregierung und Bundesbank maßgebend bei der Forderung nach der Unabhängigkeit der Zentralbank, der Festlegung von Preisstabilität als vorrangiges Ziel, der Festlegung der Konvergenzkriterien für den Beitritt zum Euro, der Einfügung der No-bail-out-Klausel (Art. 125 Vertrag über die Arbeitsweise der Europäischen Union, AEUV), der Übertragung der Verantwortung für die Fiskalpolitik auf die Mitgliedstaaten im Rahmen des Verfahrens bei einem übermä-

22 Alle Zahlen basieren auf Eurostat-Daten. Für eine ausführlichere Diskussion siehe Bulmer/Paterson: Germany and the European Union, 2019, S. 61ff.

23 Konrad Popławski: The role of Central Europe in the German economy. The political consequences, Centre for Eastern Studies: OSW Report September 2016, S. 5.

24 Stockholm International Peace Research Institute (Hrsg.): Military expenditure by country as percentage of gross domestic product, 1988-2002, 2017, abrufbar unter: www.sipri.org/sites/default/files/Milex-share-ofGDP.pdf (letzter Zugriff: 21.1.2019).

25 Simon Bulmer: Shaping the Rules? The Constitutive Politics of the European Union and German Power, in: Peter Katzenstein (Hrsg.): Tamed Power: Germany in Europe, Ithaca 1997, S. 49ff., hier S. 73f.

26 Monika Bösche: Trapped Inside the European Fortress? Germany and European Union Asylum and Refugee Policy, in: Gunther Hellmann (Hrsg.): Germany's EU Policy on Asylum and Defence. De-Europeanization by Default?, Basingstoke 2006, S. 29ff., hier S. $49 \mathrm{ff}$. 
ßigen Defizit (Art. 126 AEUV) und dem Verbot der monetären Staatsfinanzierung der Mitgliedstaaten des Euro-Währungsgebiets (Art. 123 AEUV) ${ }^{27}$ Dieses Maßnahmenbündel legte somit im Voraus die Bandbreite der Reformmöglichkeiten in Bezug auf die deutschen „sound money“-Präferenzen während der Krise in der Eurozone fest.

Tatsächlich geriet das Eintreten für mehr Integration, ein fortwährendes deutsches Leitmotiv, während der Krise in der Eurozone in ein Spannungsverhältnis zu den Prinzipien des „sound money“, die seit ihrer Gründung 1949 ein weiteres konstantes Leitmotiv der Bundesrepublik waren. Das Ergebnis war, dass weitere notwendige Integrationsschritte in der Fiskalpolitik zur Stärkung der Widerstandsfähigkeit der Eurozone ausdrücklich davon abhängig gemacht wurden, dass die Mitgliedstaaten ihre Haushaltsprobleme selbst und im Einklang mit dem Ansatz des „sound money“ zu lösen hatten. Die Ausrichtung auf diese zwei deutschen Politikgrundsätze hatte zur Folge, dass die Lasten der Anpassung den Schuldnerstaaten auferlegt wurden.

Weitere Konstanten in der deutschen Europapolitik sind der Ansatz einer „zivilen Macht" in der Außenpolitik, einschließlich der Einhaltung internationaler Rechtsgrundsätze und der Achtung der Menschenrechte. ${ }^{28}$ Dieser Ansatz passte gut zum außen- und sicherheitspolitischen Rahmen der EU, der die Verteidigungspolitik bis in die 2000er Jahre weitgehend der NATO überließ.

\section{Einschränkende Faktoren}

An dieser Stelle ist es wichtig, Gegenkräfte zu berücksichtigen. Denn es ist eine vereinfachende Annahme, dass Deutschland aufgrund von Größe, struktureller Wirtschaftskraft und ideellen Ressourcen automatisch die Rolle des hegemonialen Stabilisators der EU spielt. Innerhalb der EU gibt es wichtige systemische Grenzen, die es verhindern, dass ein einzelner Mitgliedstaat die Art von hegemonialer Rolle übernimmt, welche die Vereinigten Staaten während des zwanzigsten Jahrhunderts innehatten. So lässt beispielsweise der institutionelle Aufbau der EU eine solche Rolle eigentlich nicht zu, was insbesondere in der starken Betonung des Konsenses in der Arbeitsweise des Europäischen Rates, der „supreme political authority“ der EU, zum Ausdruck kommt. ${ }^{29}$ Abgesehen vom Europäischen Rat lässt das politische System der EU wenig Spielraum für einen einzelnen Staat, die Führungsrolle zu übernehmen. Dies ist auf die Abstimmungsverfahren im Rat und auf das Gesetzgebungsverfahren seit Lissabon zurückzuführen.

Politische Zwänge verbinden sich mit systemischen, wodurch Legitimitätsbedenken konkret zum Ausdruck gebracht werden: Sei es der Widerstand Ungarns gegen das von Deutschland bevorzugte Umverteilungssystem für Flüchtlinge oder die Opposition südeuropäischer PolitikerInnen und BürgerInnen gegen die Sparpolitik, für die sie Deutschland während der Krise in der Eurozone verantwortlich machten. Letztere äußerte sich sogar in spezifischer antideutscher Stimmung bei öffentlichen Demonstrationen. Das Besondere an der politischen Legitimierung der letzten Jahre ist, dass der latente Widerstand gegen einen dominanten Staat nicht mehr auf Partnerregierungen beschränkt ist, sondern sich auf-

27 Bulmer/Paterson: Germany and the European Union, 2019, S. 173

28 Vgl. Hanns Maull: Foreign policy: from 'civilian power' to 'trading state'?, in: Sarah Colvin (Hrsg.): Routledge Handbook of German Politics \& Culture, Abingdon/New York 2015, S. 409ff.

29 Fiona Hayes-Renshaw/Helen Wallace: The Council of Ministers, 2. Auflage, Basingstoke 2006, S. 1. 
grund der Mobilisierung euroskeptischer Kräfte, unterstützt durch die sozialen Medien, schnell auf die breite Öffentlichkeit ausweiten kann.

Ungeachtet des deutschen Regierungshandelns ist klar, dass die erhebliche Ressourcenmacht Deutschlands auf Gegenkräfte stößt, die sich aus dem EU-System sowie potenziell aus themenbezogenen Bedenken der EU-Partner ergeben. Solche Bedenken können durch gemeinsame Initiativen abgebaut werden, wie es in der Vergangenheit vielfach mit Frankreich der Fall war. Andere bilaterale Partnerschaften, wie beispielsweise mit Polen (einschließlich des Weimarer Dreiecks), haben sich angesichts der Verschlechterung der Beziehungen nach dem Wahlsieg der Partei „Recht und Gerechtigkeit“ Ende 2015 als weniger dauerhaft erwiesen. So waren z.B. die Äußerungen des polnischen Außenministers nach der Wiederwahl (mit deutscher Unterstützung) von Donald Tusk zum Präsidenten des Europäischen Rates, einem ehemaligen innenpolitischen Konkurrenten, umstritten: „We know now that it [the EU] is a union under Berlin's diktat. “30 Die Verschlechterung des Verhältnisses zu Polen, dem größten Staat der V4, hat wiederum zu schwierigen Beziehungen zu den mitteleuropäischen Staaten geführt. Allen voran steht der ungarische Ministerpräsident Viktor Orbán, der die Opposition gegen die Flüchtlingspolitik von Bundeskanzlerin Merkel im Jahr 2015 anführte.

Allgemeiner formuliert: Die größere Vielfalt der Interessen in der derzeitigen EU in Verbindung mit dem stark spaltenden Diskurs, der mit dem Aufstieg der euroskeptischen Kräfte einherging, erschwert es einzelnen Mitgliedstaaten, die Rolle des hegemonialen Stabilisators einzunehmen. Hinzu kommt, dass die deutsch-französische Partnerschaft nicht mehr das Spektrum der politischen Stimmung der gesamten EU abdeckt, wie es in früheren Jahrzehnten der Fall war. Die Vielfalt der politischen Ansichten ist heute komplexer. Das schließt zwar nicht aus, dass Deutschland bei bestimmten politischen Fragen möglicherweise die Rolle des Hegemonen einnimmt. Trotz seiner Position als „Macht in der Mitte" erscheint eine systemrelevante Rolle jedoch unwahrscheinlich. ${ }^{31}$

\section{Der nationale Kontext}

Diese äußeren Zwänge bezüglich der Rolle Deutschlands als Hegemon in der EU sind beträchtlich. Die zunehmende Politisierung der Europapolitik im nationalen Kontext schränkt sie noch weiter ein. Die deutsche Innenpolitik hat stets das Wirken des Landes im europäischen Integrationsprozess beeinflusst. ${ }^{32}$ Traditionell sorgte sie für einen starken „permissive consensus“ zugunsten der europäischen Integration unter den politischen Parteien, Interessengruppen und der Öffentlichkeit. Andererseits enthielt das institutionelle System, das während der Bonner Republik durch „Semisouveränität“ gekennzeichnet und mit dem starken Bekenntnis der Bundesrepublik zur deutsch-französischen Partnerschaft verbunden war, Hürden für jegliche Überlegungen bezüglich einer hegemonialen Rolle Deutschlands. Der deutsche Konsens über die europäische Integration ergab sich aus einer

30 Jennifer Rankin: Poland reacts with fury to re-election of Donald Tusk, in: The Guardian, 9. März 2017.

31 Vgl. Herfried Münkler: Macht in der Mitte. Die neuen Aufgaben Deutschlands in Europa, Hamburg 2015.

32 Simon Bulmer/William Paterson: The Federal Republic of Germany and the European Community, Abingdon/New York 2015 (Originalausgabe veröffentlicht 1987 in London). 
„politics of centrality“33 in der Parteipolitik: eine starke politische Mitte als Gegenmodell zu den Extremen aus Nazi- und DDR-Zeit.

Diese Situation vor der Wiedervereinigung hat sich in den letzten drei Jahrzehnten zu einem gewissen Grad verändert. Zum einen stärkte die Bundesregierung ihre Verfahren zur Koordinierung der Europapolitik. ${ }^{34}$ Mit der Einführung der Abteilungsleiterrunde im Jahr 2000 wurde ein wichtiges exekutives Abstimmungsgremium geschaffen, das dazu beitrug, Divergenzen zwischen den Ministerien zu antizipieren und zu reduzieren. Darüber hinaus wurden klare Leitlinien für die Politikkoordinierung innerhalb der Bundesregierung festgelegt, wie sie im EU-Handbuch über Entscheidungsprozesse, Koordinierung, Verfahren und Unterrichtungsaufgaben der Bundesregierung zu finden sind. ${ }^{35}$ In den Krisenjahren spielte auch das Bundeskanzleramt eine stärkere Rolle bei der Politikkoordinierung, insbesondere während der Krise in der Eurozone und der Flüchtlingskrise, da diese Themen auf der Tagesordnung des Europäischen Rates weit oben standen. Dennoch wird in Brüssel gelegentlich Kritik an der deutschen Ineffizienz laut. ${ }^{36}$

Innerhalb der Bundesregierung sind die Stimmen anderer am Politikprozess Beteiligter lauter geworden. Die Regierungen der Bundesländer wurden in den Bereichen gestärkt, in denen die EU-Politik ihre Zuständigkeiten mit dem Vertrag von Maastricht (überarbeiteter Art. 23 GG) berührte. Die Einführung von Konsultationsverfahren nach dem Gesetz über die Zusammenarbeit von Bund und Ländern in Angelegenheiten der Europäischen Union (EUZBLG) steckte den Rahmen für die Koordinierung ab. Tatsächlich scheinen die Beziehungen Berlins zu den Ländern bezüglich der Europapolitik seit dem Ende des Jahrzehnts der EU-Verfassungsdebatte (2000er Jahre), in dem die Landesregierungen aktiv beteiligt waren, zu funktionieren. Teilweise mag dies daran liegen, dass die Kernpunkte der EU-Krisenagenda kaum die Kompetenzen der Länder betrafen (wie z.B. die Ukraine- und Eurozonenkrise). Die Flüchtlingskrise führte jedoch zu Spannungen, die sich am stärksten durch die CSU in Bayern äußerten, da das Bundesland besonders betroffen war. Natürlich gibt es gelegentlich Differenzen zwischen Ländern und Bund, z.B. in Bezug auf die EU-Bodenschutzrichtlinie 2007. Anders als die Drohung der Landesregierungen 1992/1993, die Ratifizierung des Vertrags von Maastricht zu blockieren, wirken sich diese eher technischen Differenzen nicht auf die Rolle Deutschlands in der EU aus.

Die Situation im Bundestag stellt dazu einen gewissen Kontrast dar. Seit dem Urteil des Bundesverfassungsgerichts 1993 wurden dessen Kompetenzen mehrfach erweitert, so z.B. mit der Entscheidung bezüglich der Einführung einer einheitlichen Währung. Aufgrund der überwiegend integrationsfreundlichen Haltung der Parteien kamen diese ersten zusätzlichen Befugnisse infolge einer Klage vor dem Bundesverfassungsgericht hinzu, wobei das Gericht in seiner Verantwortung für die Wahrung des demokratischen Kernbestands des Grundgesetzes in Deutschland handelte. Änderungen des Grundgesetzes (Art. 23 und 45) und die Einführung des Gesetzes über die Zusammenarbeit von Bundesregierung und

33 Gordon Smith: Democracy in Western Germany: Parties and Politics in the Federal Republic, London 1979, S. 213f.

34 Bulmer/Paterson: Germany and the European Union, 2019, S. 92ff.; Timm Beichelt: Over-efficiency in German EU policy coordination, in: German Politics 4/2007, S. $421 \mathrm{ff}$.

35 Bundesministerium für Wirtschaft und Energie: EU-Handbuch: Entscheidungsprozesse, Koordinierung, Verfahren und Unterrichtungsaufgaben der Bundesregierung, 12. Ausgabe, Berlin 2014, S. $121 \mathrm{ff}$.

36 Z.B. Christoph Schiltz: Die sieben Todsünden der Deutschen in Brüssel, in: WELT, 23. November 2007. 
Deutschem Bundestag in Angelegenheiten der Europäischen Union (EUZBBG) 1993 schufen einen neuen Rahmen. ${ }^{37}$ Mit dem Gesetz über die Wahrnehmung der Integrationsverantwortung des Bundestages und des Bundesrates in Angelegenheiten der Europäischen Union (IntVG) aus dem Jahr 2009 kamen nach dem Lissabon-Urteil des Bundesverfassungsgerichts neue Kontrollaufgaben hinzu. Schließlich wurde das EUZBBG mehrfach überarbeitet, u.a. infolge verschiedener Urteile des Bundesverfassungsgerichts, wodurch die parlamentarische Kontrolle während der Krise in der Eurozone gestärkt wurde. ${ }^{38}$

Obwohl es manchmal den Anschein erweckte, als wären dem Bundestag Kompetenzen übertragen worden, die er eigentlich nicht wollte, hat sich dessen Einbringung in EU-Angelegenheiten seit 2007 gewandelt. Dies zeigt sich insbesondere durch eine verstärkte administrative Unterstützung, um den neuen Kompetenzen gerecht zu werden. Die besondere Bedeutung der Reformen liegt aber darin, dass der Bundestag dadurch sogar zu einem Vetospieler für die Europapolitik werden kann. Während der zweiten Koalitionsregierung unter Merkel (aus CDU, CSU und FDP; von 2009 bis 2013) war es für einige Maßnahmen in der Eurozone nicht möglich, eine „Kanzlermehrheit“ zu erhalten. Man war auf die Stimmen der Bundestagsabgeordneten aus der Opposition angewiesen. ${ }^{39}$ Mit dem zerfallenden parteipolitischen Konsens und dem Einzug der Alternative für Deutschland (AfD) in den Bundestag als größte Oppositionspartei wird es wahrscheinlicher, dass diese Kräfte die Politik der Bundesregierung erschweren - auch wenn dies bis jetzt noch nicht der Fall ist.

Das Eingreifen des Bundesverfassungsgerichts in Belange der Europapolitik in der Zeit nach dem Vertrag von Maastricht war für die Entwicklung der Zuständigkeiten im Bundesrat und Bundestag wesentlich. Die Rolle des Bundesverfassungsgerichts bestand darin, das parlamentarische System gegen Eingriffe der EU zu verteidigen. Bereits im Jahr 2009 konnte man feststellen, dass dem Bundesverfassungsgericht eine zunehmende Bedeutung in der Europapolitik der Bundesregierung zukommt. ${ }^{40}$ Dies wurde während der Krise in der Eurozone offensichtlich, als eine Reihe von verfassungsrechtlichen Bedenken die Zukunft der Eurozone zeitweise von Urteilen aus Karlsruhe abhängig zu machen schien. Letzten Endes kam es zu keinem Blockade-Urteil. Gleichwohl wurden EU-BeobachterInnen die Einschränkungen der deutschen Regierungspolitik durch interne Schranken klarer.

Ein weiterer institutioneller Aspekt wurde während der Krise in der Eurozone wieder sichtbar, nämlich das Potenzial der Bundesbank als scharfer Kritiker der Europapolitik. Die Aufgabe des Präsidenten Jens Weidmann war es sicherzustellen, dass die Europäische Zentralbank (EZB) den Vorgaben folgte, die Weidmanns Vorgänger in der Bundesbank bei den Verhandlungen über die Satzung der EZB gemacht hatten. Seine Stimme war unter den KritikerInnen der Eurozone und der Geldpolitik der EZB laut und deutlich zu vernehmen und stellt damit eine weitere Quelle der Politisierung der Europapolitik dar.

37 Sven Hölscheidt: The German Bundestag: From Benevolent 'Weakness' Towards Supportive Scrutiny, in: Andreas Maurer/Wolfgang Wessels (Hrsg.): National Parliaments on their Ways to Europe: Losers or Latecomers?, BadenBaden 2001, S. 117ff., hier S. 118.

38 Bulmer/Paterson: Germany and the European Union, 2019, S. 97f.; Timm Beichelt: Deutschland und Europa. Die Europäisierung des politischen Systems, 2. Auflage, Wiesbaden 2015.

39 Andreas Wimmel: Fachliche Expertise und abweichendes Verhalten bei Abstimmungen zur Euro-Krise im Deutschen Bundestag, in: Zeitschrift für Politikberatung 3-4/2013, S. 125ff.

40 Peter Becker/Andreas Maurer: Deutsche Integrationsbremsen. Folgen und Gefahren des Karlsruher Urteils für Deutschland und die EU, Stiftung Wissenschaft und Politik: SWP-Aktuell 41/2009. 
Die parteipolitische Landschaft in Deutschland hat sich in der Zeit nach der Wiedervereinigung stark verändert. Seit 1990 hat sich das politische Spektrum von einem 2,5- zu einem 6-Parteien-System gewandelt. Die euroskeptischen Parteien im Bundestag - DIE LINKE und die AfD - haben bei der Bundestagswahl 2017 zusammen einen Anteil von 21,8 Prozent der Zweitstimmen erreicht: eine markante Entwicklung. Dies erschwerte nicht nur die Koalitionsbildung, wie die sechs Monate nach der Bundestagswahl gezeigt haben, sondern stärkt auch die politischen Kräfte, welche sich die institutionelle Anreizstruktur nach Maastricht im Sinne eines machtvolleren Bundestags zu Nutze machen können.

Meinungsverschiedenheiten zwischen einzelnen Bundestagsabgeordneten und unter den Parteien in Bezug auf die Krise in der Eurozone und die Flüchtlingskrise haben ebenso zur Politisierung der deutschen Europapolitik beigetragen. Darüber hinaus sind die Verhandlungen über eine „Jamaika-Koalition“ aus CDU, CSU, FDP und Bündnis 90/Die Grünen angeblich gescheitert, weil die FDP mit den Inhalten zur Eurozone unzufrieden war. ${ }^{41}$ Ein weiteres Risiko besteht derzeit durch den nachlassenden Wählerzuspruch für die Sozialdemokraten, wie die Wahlen in Bayern und Hessen verdeutlichten. Sollte die SPD beschließen, die Große Koalition zu verlassen, in der Hoffnung, sich dadurch wieder mehr Unterstützung bei den WählerInnen zu sichern, würde dies die Autorität der Regierung schwächen, bis eine stabilere Koalition gebildet werden könnte.

Die Politisierung der Parteienlandschaft geht einher mit einem zunehmenden Einfluss der Öffentlichkeit auf die Europapolitik. Sowohl bei der Krise in der Eurozone als auch bei der Flüchtlingskrise musste die öffentliche Meinung berücksichtigt werden. Der Unterschied bei den beiden Krisen bestand darin, dass sich Bundeskanzlerin Merkel und ihre Regierung bei der Krise in der Eurozone mit der öffentlichen Meinung im Einklang befanden, während Berlin in der Flüchtlingskrise vorpreschte und so rechts von CDU/CSU Platz im politischen Spektrum schuf. Ein wichtiger Prüfstein für den Tenor der nationalen Europadebatte werden die Wahlen zum Europäischen Parlament im Mai 2019 sein.

Interessengruppen sind nach wie vor maßgeblich an der Gestaltung der Politik der Bundesregierung beteiligt, unter anderem in Bezug auf spezifische Politiken zu Handels- und Umweltschutzvorschriften. In der Vergangenheit war der parteipolitische Konsens über die EU für sie von Nutzen. Sie konnten Einfluss auf die Europapolitik der Regierung nehmen, ohne den Parteien Aufmerksamkeit schenken zu müssen. Die Chemie- und die Automobilindustrie verstanden ihr Lobbying meisterlich in Bezug auf die EU-Gesetzgebung. Interessant war jedoch, dass in der politischen Debatte über die Transatlantische Handels- und Investitionspartnerschaft (TTIP) die Stimme der Wirtschaft, insbesondere des Bundesverbandes der Deutschen Industrie (BDI), erhebliche Schwierigkeiten hatte, bei einem Handelsabkommen, das für deutsche Exporteure ein beträchtliches Potenzial zu bieten schien, Zugkraft zu entwickeln. Letztendlich beendete Trump die Verhandlungen, aber vieles deutete darauf hin, dass auch deutsche Wirtschaftseliten der allmählichen Politisierung der Europapolitik ausgesetzt sein könnten.

All diese Entwicklungen - sowohl innerhalb der EU als auch innerhalb Deutschlands zeigen, dass es erhebliche institutionelle und politische Einschränkungen innerhalb der EU und Deutschlands gibt, die einer hegemonialen Rolle Deutschlands entgegenstehen.

41 Cerstin Gammelin: Wie die FDP die Verhandlungen zur Europapolitik platzen ließ, in: Süddeutsche Zeitung, 23. November 2017. 
Nichtsdestotrotz ist zu prüfen, ob Deutschland diese Rolle durch sein Handeln nicht doch einnimmt.

\section{Die deutsche Gestaltungsmacht in den Krisenjahren}

Die EU wurde in den 2010er Jahren aufgrund der Ereignisse zu einer Politik des Krisenmanagements gezwungen. ${ }^{42} \mathrm{Zu}$ Beginn des Jahrzehnts erreichte die Krise in der Eurozone ihren Höhepunkt. 2014/2015 spitzte sich die Ukraine-Krise ebenso wie die Flüchtlingskrise zu. Die britische Entscheidung, die EU zu verlassen, wurde mit dem Referendum im Juni 2016 besiegelt. Wenn Großbritannien aus der EU austritt, bleibt dies nicht ohne Folgen für die Stellung der EU in der Welt, insbesondere bezogen auf sicherheits- und verteidigungspolitische Fragen. Ungeachtet dieser Krisen - und konfrontiert mit Angriffen auf die Rechtsstaatlichkeit in Mitteleuropa - setzt die EU ihr politisches Tagesgeschäft fort.

Eine Entwicklung ist jedoch offensichtlich geworden: Die Integrationspolitik ist während dieser Zeit in den Hintergrund der deutschen Europapolitik getreten und nationale Interessen stehen infolgedessen mehr im Vordergrund. Die Arbeit von Bundeskanzlerin Merkel in den 2000ern, den Verfassungsvertrag wiederzubeleben und die Verabschiedung des Vertrags von Lissabon zu ermöglichen, sollte dennoch nicht unterschätzt werden. In den 2010er Jahren beschränken sich die Integrationsvorhaben dann auf spezifische politische Entwicklungen, insbesondere in Bezug auf Reformen in der Eurozone oder die Stärkung der Sicherheitspolitik (durch die Ständige Strukturierte Zusammenarbeit, SSZ; engl. Permanent Structured Cooperation, PESCO) angesichts des Brexits. Eine umfassendere Vision für die europäische Integration fehlt.

Der EU-Erweiterung fühlen sich seit jeher deutsche Bundesregierungen verpflichtet. Allerdings ist Deutschland auch in diesem Politikbereich weniger proaktiv, was vor allem auf die fehlende Bereitschaft von Bewerbern zum EU-Beitritt (abgesehen von Kroatien) zurückzuführen ist. Die deutsche „Doppelstrategie“ der Erweiterung und Vertiefung, die darauf abzielt, die EU nicht durch den Beitritt neuer Mitgliedstaaten zu schwächen, ${ }^{43}$ wurde insofern ausgesetzt. Zugleich setzt die Bundesregierung das traditionelle deutsche Engagement der Förderung von Frieden, Versöhnung und Demokratie in Europa auf dem Westbalkan fort, auch wenn die Wirkung dieser Politik weniger deutlich wird. ${ }^{44}$

In einigen anderen Politikbereichen versuchte Deutschland, eine Führungsrolle einzunehmen, etwa in den ersten Jahrzehnten der Umweltpolitik oder beim Klimawandel. Doch dieser Versuch ist aufgrund der andauernden Nutzung von Kohle (wegen des Ausstiegs aus der Kernenergie) und in Verbindung mit dem Dieselskandal nicht mehr ganz überzeugend. Die Automobilindustrie ist ein klassisches Beispiel dafür, wie die dort vorhandene wirtschaftliche Strukturmacht in das Regierungshandeln einfließt. So gab es nach dem Die-

42 Vgl. Desmond Dinan/Neill Nugent/William Paterson (Hrsg.): The European Union in Crisis, London 2017.

43 Vgl. Barbara Lippert: Die Bundesrepublik Deutschland und die Erweiterung der Europäischen Union, in: Katrin Böttger/Mathias Jopp (Hrsg.): Handbuch zur deutschen Europapolitik, Baden-Baden 2016, S. 393ff., hier S. $398 \mathrm{ff}$.

44 Katrin Böttger/Mathias Jopp: Grundlinien deutscher Europapolitik, in: Katrin Böttger/Mathias Jopp (Hrsg.): Handbuch zur deutschen Europapolitik, Baden-Baden 2016, S. 13ff., hier S. 23. 
selskandal mehr Treffen der Regierung mit der Automobilindustrie als mit Umweltverbänden. ${ }^{45}$

Beim mehrjährigen Finanzrahmen der EU ist die Regierung typischerweise zwischen verschiedenen Interessen hin- und hergerissen: Sparen (Finanzministerium), Integrationspolitik (Auswärtiges Amt), Ausgaben (ausgabentätigende Ministerien und Regierungen der Länder), sodass eine klare politische Führung beeinträchtigt sein kann. ${ }^{46}$ Deutschland profitiert überraschenderweise besonders stark von den Europäischen Struktur- und Investitionsfonds und erhält mehr als 16 Prozent der EU-Mittel für den Zeitraum 2014 bis $2020 .{ }^{47}$

In vielen anderen Bereichen sind die politischen Entwicklungen inkrementell und erfolgen im Rahmen des ordentlichen Gesetzgebungsverfahrens, in dem der Einfluss Deutschlands geringer ist. Es ist also wichtig, alle Arten von Politikbereichen zu beachten, wenn man die Gestaltungsmacht Deutschlands bespricht. Diese ist zwangsläufig dort umstrittener, wo die Interessen der Staaten gegeneinander ausgespielt werden.

Im Folgenden wird nun die deutsche Gestaltungsmacht am Beispiel der Krise in der Eurozone sowie der Ukraine- und Flüchtlingskrise - zugegebenermaßen knapp - analysiert. ${ }^{48}$ Während der Krise in der Eurozone war die deutsche Politik von folgenden Grundsätzen geprägt: ${ }^{49}$

- Schuldnerstaaten sollten Konsequenzen aus der Krise ziehen und die notwendigen innenpolitischen Reformen umsetzen.

- Das Konzept der Wirtschafts- und Währungsunion aus dem Vertrag von Maastricht musste korrigiert werden, damit sich solche Situationen nicht wiederholen konnten (strengere Fiskalregeln).

- Eine Bankenunion wurde benötigt, um der Eurozone mehr Widerstandsfähigkeit zu verleihen.

- Rettungsfonds waren als Schutzmechanismus und zur Gefahrenabwehr notwendig.

$\mathrm{Zu}$ Beginn der Krise in der Eurozone im Frühjahr 2010 nahm Deutschland die Rolle des zögerlichen Hegemonen ein. ${ }^{50}$ Aus Sorge vor einem „Moral Hazard“ wollte es nicht zu früh eingreifen. Letztlich musste die Krise erst das System gefährden, bevor Deutschland handelte. Kanzlerin Merkel setzte sich über die Vorschläge von Finanzminister Wolfgang Schäuble für einen Europäischen Währungsfonds hinweg und bestand auf einer Lösung,

45 Christina Deckwirth: Lobbykontakte: Bundesregierung bevorzugt die Autoindustrie, in: Lobby Control, 14. September 2017.

46 Peter Becker: Zwischen Zuchtmeister und Zahlmeister - Deutsche Europapolitik und die europäischen Finanzverhandlungen, in: Katrin Böttger/Mathias Jopp (Hrsg.): Handbuch zur deutschen Europapolitik, BadenBaden 2016, S. 217ff.

47 Europäische Kommission: Europäische Struktur- und Investitionsfonds - Deutschland, April 2016; Bundesministerium für Wirtschaft und Energie: Die Europäischen Struktur- und Investitionsfonds in Deutschland, Berlin 2017.

48 Siehe auch Simon Bulmer/William Paterson: Deutschlands Rolle bei der Bewältigung der europäischen Währungs- und Migrationskrisen, in: Werner Weidenfeld/Wolfgang Wessels (Hrsg.): Jahrbuch der Europäischen Integration 2016, Baden-Baden 2016, S. 43ff.; Joachim Schild: The Myth of German Hegemony in the Euro Area Revisited, in: West European Politics, im Erscheinen.

49 Ausführlichere Informationen hierzu siehe Bulmer/Paterson: Germany and the European Union, 2019, S. $167 \mathrm{ff}$.

50 William Paterson: The Reluctant Hegemon? Germany Moves Centre Stage in the European Union, in: Journal of Common Market Studies s1/2011, Special Issue: The JCMS Annual Review of the European Union in 2010, S. $57 \mathrm{ff}$. 
bei der der Internationale Währungsfonds Teil einer „Troika“ zur Beaufsichtigung der griechischen Rettung sein sollte. Deutschland spielte aus mehreren Gründen die Rolle des zögerlichen Hegemonen: Es existierte kein auf das vorhandene Problem anwendbares Verfahren; ordoliberal inspirierten Prinzipien zufolge musste Griechenland die Verantwortung für seine Lage selbst übernehmen; und wegen ihrer knappen Mehrheit im Bundestag und der Unsicherheit hinsichtlich der Haltung der Sozialdemokraten (sie enthielten sich weitgehend) musste die Bundesregierung vorsichtig sein. Deutschland beharrte darauf, dass die Rettung durch die EU von griechischen Wirtschaftsreformen abhing.

Bei der Neugestaltung des Systems der Eurozone war Deutschland mit seinen Vorstellungen zur Stärkung der Haushaltsüberwachung maßgeblich. Orientiert am Ordoliberalismus konnte Deutschland mit Unterstützung des französischen Präsidenten Nicolas Sarkozy die Führungsrolle übernehmen. Die im Fiskalpakt enthaltenen Maßnahmen zur Verschärfung der Überwachung, wie die Regel zum ausgeglichenen Haushalt, die Schuldenbremse und der Gesetzescharakter, den diese Regeln mindestens haben müssen, fußen auf diesen ordoliberalen Prinzipien. Deutschland selbst hatte 2009 die sogenannte Schuldenbremse eingeführt (Art. 109 GG). Im Gegensatz dazu scheiterten andere Optionen, die eine Konsolidierung der Schulden der Eurozone mit sich gebracht hätten, wie z.B. Eurobonds oder jegliche Art von „Transferunion“, am deutschen Veto. Das Zugeständnis an Frankreich bestand darin, dass der Europäische Rat die von der französischen Regierung gewünschte Rolle der „Economic Governance“ übernehmen konnte. Ein inhaltlicher Kurswechsel Deutschlands war damit allerdings nicht verbunden.

Bei der Stärkung der Haushaltsüberwachung erwiesen sich deutsche Ideen als einflussreich, ja sogar hegemonial. ${ }^{51}$ Dass sie zum Tragen kamen wurde durch die asymmetrischen Machtverhältnisse zwischen Gläubiger- und Schuldnerstaaten erleichtert. Kritik an den von Deutschland inspirierten „Sparpolitik“-Maßnahmen kam aus südeuropäischen Staaten wie Griechenland und Spanien. Dullien und Guérot äußerten sich dazu wie folgt: „Germany has been widely criticised for its monetary policy, its inflexibility on austerity measures, its rigid legal approach to treaty change and its selfish view of trade imbalances. ${ }^{\text {"5 }}$ Diese Staaten befanden sich jedoch in einer schwachen Verhandlungsposition. Der Druck aus der deutschen Innenpolitik führte dazu, dass die Politik des „sound money“ durchgesetzt wurde. Die Politisierung der öffentlichen Meinung, der Druck der Bundesbank, die parlamentarische Kontrolle und die Gefahr eines negativen Urteils des Bundesverfassungsgerichts waren hierfür ausschlaggebend.

Im Gegensatz dazu spielte Deutschland bei der Bankenunion keine vergleichbare Rolle. Zwar gelang es sicherzustellen, dass der Einheitliche Abwicklungsfonds bei Bankeninsolvenzen nicht dazu führte, dass zusammenbrechende ausländische Banken mit deutschen Mitteln unterstützt werden. In anderen Fragen war Deutschland jedoch aufgrund unterschiedlicher Interessen inländischer Banken und Positionen innerhalb der Bundesregierung weniger einflussreich. ${ }^{53}$

51 Siehe auch Magnus Schoeller: Providing political leadership? Three case studies on Germany's ambiguous role in the eurozone crisis, in: Journal of European Public Policy 1/2017, S. 1ff., hier S. 12.

52 Sebastian Dullien/Ulrike Guérot: The Long Shadow of Ordoliberalism: Germany's Approach to the Euro Crisis, European Council on Foreign Relations: Policy Brief 49/2012.

53 Siehe Bulmer/Paterson: Germany and the European Union, 2019, S. 190ff.; David Schäfer: A Banking Union of Ideas? The Impact of Ordoliberalism and the Vicious Circle on the EU Banking Union, in: Journal of Common 
Festzuhalten bleibt also, dass es die hegemonialen Ideen von Ordoliberalismus und „sound money“ waren, die auf deutscher Seite einflussreich waren. Deutschland spielte nicht die Rolle des hegemonialen Stabilisators und schon gar nicht in der von Kindleberger vorgesehenen klassischen Weise. Denn es bot weder einen Markt für „distressed goods“, die Möglichkeit der antizyklischen Kreditvergabe oder des „lending of last resort“ noch die der makroökonomischen Koordinierung. ${ }^{54}$ Stattdessen war es eher das Engagement des Präsidenten der Europäischen Zentralbank, Mario Draghi, „to do whatever it takes to preserve the euro" ${ }^{\text {" } 55}$ das die Finanzmärkte endgültig beruhigte.

Im Falle der Ukraine-Krise waren die Umstände etwas anders. Die militärischen Ressourcen Deutschlands entsprachen nicht den wirtschaftlichen, über die das Land im Zusammenhang mit der Krise in der Eurozone verfügte. Aus historischen Gründen ist die Vorstellung des Einsatzes militärischer Mittel in der internationalen Politik sowohl für die politischen EntscheidungsträgerInnen als auch für die öffentliche Meinung ein Gräuel. Außerdem stellt das von Deutschland verfolgte Leitmotiv einer Zivilmacht den Einsatz von Politik und Diplomatie vor den Einsatz von Gewalt. Bundeskanzlerin Merkel übernahm die führende Rolle, andere EU-Staaten davon zu überzeugen, die Einhaltung internationaler Rechtsgrundsätze in den Mittelpunkt der Politik zu stellen und Wirtschaftssanktionen zu verhängen. Der Abschuss des malaysischen Flugzeugs MH17 über der Ostukraine hat dazu beigetragen, dass die EU-Partner Merkels Politik unterstützten. Deutschland selbst musste durch die Umsetzung der Sanktionspolitik erhebliche Einbußen im Handel hinnehmen. Das sogenannte Normandie-Format für wichtige Gespräche mit Russland und der Ukraine ermöglichte der deutsch-französischen Partnerschaft eine Schlüsselrolle auf der internationalen Bühne. In diesem Fall übernahm die deutsche Diplomatie eine Führungsaufgabe und der Ansatz der zivilen Macht war ein wichtiger Ideenlieferant. Jenseits dieser Bedeutung Deutschland als Hegemon zu bezeichnen, erscheint aufgrund der begrenzten militärischen Ressourcen problematisch, ganz zu schweigen von den pazifistischen Tendenzen in der deutschen Öffentlichkeit.

Das Beispiel der Flüchtlingkrise ist wiederum anders gelagert. Kanzlerin Merkels Ausspruch „Wir schaffen das“ Ende August 2015 legte die Vermutung nahe, dass Deutschland angesichts der humanitären Katastrophe die Rolle von „Europe’s lonely liberal hegemon“"56 übernimmt. Doch diese Position geriet Anfang 2016 durch zwei Entwicklungen ins Wanken: Erstens widersetzten sich die Tschechische Republik, Ungarn, Rumänien und die Slowakei den EU-Maßnahmen, die mit qualifizierter Mehrheit im Ministerrat verabschiedet worden waren. Der Wunsch Deutschlands und der Europäischen Kommission nach einer Umverteilung der Flüchtlinge wurde daher auf Eis gelegt. Zweitens schwand die Unterstützung für Merkels Politik in der deutschen Öffentlichkeit, da das Ausmaß der Kosten für die Integration von Flüchtlingen immer deutlicher wurde. Letztendlich erwies sich die „cash

Market Studies 4/2016, S. 961ff.; Rachel Epstein/Martin Rhodes: International in Life, National in Death? Banking Nationalism on the Road to Banking Union, Kolleg-Forschergruppe „The Transformative Power of Europe“: KFG Working Paper 61/2014.

54 Matthias Matthijs/Mark Blyth: Why Only Germany Can Fix the Euro. Reading Kindleberger in Berlin, in: Foreign Affairs, 17. November 2011.

55 Europäische Zentralbank: Speech by Mario Draghi, President of the European Central Bank at the Global Investment Conference in London 26 July 2012.

56 Thorsten Benner: Europe's lonely liberal hegemon. Angela Merkel's migration policy isn't driven by morality, in: Politico, 2. Juni 2016. 
for cooperation“-Politik mit der Türkei als einzige Lösung, die steigende Anzahl von Flüchtlingen in die EU zu stoppen und mit dieser Strategie einer „Auslagerung“ des Problems eine Schadensbegrenzung der ursprünglichen Willkommenspolitik zu erreichen. In den Verhandlungen mit der Türkei spielte Bundeskanzlerin Merkel eine Schlüsselrolle. Von einer hegemonialen Stabilisatorrolle kann bei der Flüchtlingsproblematik aber nicht die Rede sein, da sich die mitteleuropäischen Mitgliedstaaten der deutschen Politik entgegenstellten.

\section{Eine eingeschränkte hegemoniale Rolle}

Aus dem Vorgesagten folgt, dass die Beweise für die deutsche Hegemonie recht begrenzt sind. Deutschland verfügt zwar über eine beträchtliche strukturelle Wirtschaftsmacht und die notwendigen Ressourcen, um die Rolle des hegemonialen Stabilisators zu spielen. Es hat sich jedoch damit auseinanderzusetzen, dass die EU prinzipiell als ein „,anti-leadership environment" konzipiert wurde, wo kein Mitgliedstaat uneingeschränkt herrschen kann. ${ }^{57}$ Die drei Krisen fanden unter Umständen statt, für die die EU-Verträge keine klaren Verfahren angeboten haben. Daher waren „emergency politics“ im Europäischen Rat nötig, was den potenziellen Einfluss der deutschen Regierung und Bundeskanzlerin Merkels erhöht hat.

Von den drei Fällen zeigte die Flüchtlingskrise deutlich, dass einer deutschen Hegemonie klare Grenzen gesetzt waren. Die mitteleuropäischen Mitgliedstaaten blockierten die deutsche Politik. Die Rolle in der Krise in der Eurozone kann noch am ehesten als hegemonial interpretiert werden, wobei die Anzeichen hierfür je nach Teilaspekt der Krise unterschiedlich waren: anfängliche Zurückhaltung im Jahr 2010, Hegemonie hinsichtlich Ideen für die Reform des Systems der Haushaltsüberwachung, aber weniger Einfluss auf Teile der Bankenunion. Im Falle der Ukraine-Krise scheint die Zuschreibung „Hegemonie“ weniger passend. Deutschland übernahm zwar eine wichtige Führungsrolle bei der Suche nach einer Lösung auf Grundlage der Einhaltung des Völkerrechts und von Sanktionen. ${ }^{58}$ Die EU sah sich jedoch mit der Unerbittlichkeit der Politik von Präsident Wladimir Putin konfrontiert, einschließlich der Annexion der Krim. Tatsachen wie diese oder die Abhängigkeit von Präsident Recep Tayyip Erdoğan, den Flüchtlingsstrom zu stoppen, verdeutlichen, wie ein verändertes internationales Umfeld auf die deutsche Europapolitik einwirkt.

Anders als die „Krisenpolitik“ wird eine Vielzahl anderer EU-Politiken routinemäßig behandelt. In diesen Fällen ist es problematisch, Argumente für eine deutsche Hegemonie zu finden, da die EU darauf ausgerichtet ist, eine solche Situation gerade zu vermeiden. Genau genommen scheint das Potenzial deutscher Hegemonie am größten, wenn die bestehende EU-Politik kein geregeltes Verfahren bietet, wenn Krisenverhältnisse herrschen und wenn sich die Politikgestaltung auf den Europäischen Rat konzentriert. In solchen Situationen der Krisenpolitik sind auch politische Meinungsverschiedenheiten zwischen den Mitgliedstaaten von solcher Bedeutung, dass die Legitimität der deutschen Hegemonie in Frage gerät, wie im Falle der Eurozonenkrise. Insgesamt sind die Aussichten auf eine deut-

57 Ludger Helms: Introduction: Leadership Questions in Transnational European Governance, in: European Political Science 1/2017, S. 1ff., hier S. 2.

58 Siehe auch Magnus Schoeller: Tracing Leadership: The ECB’s "Whatever It Takes" and Germany in the Ukraine Crisis, in: West European Politics, im Erscheinen. 
sche Hegemonie, ob im positiven oder negativen Sinn, eher begrenzt und am wahrscheinlichsten im Falle eines Wiederauflebens der Krise in der Eurozone.

Auch in Bezug auf die Frage, wie sich die deutsche Innenpolitik auf eine hegemoniale Rolle Deutschlands auswirkt, ist die Bilanz gemischt. Klar ist, erstens, dass den parlamentarischen Institutionen in der Zeit nach Maastricht mehr Kompetenzen übertragen wurden. Darüber hinaus ist die politische Bühne in Bezug auf die Europapolitik immer umkämpfter. Es stellt sich daher die Frage, ob diese beiden Entwicklungen die Politik der Bundesregierung einschränken oder erleichtern. Im Falle der Krise in der Eurozone stützten sie die politische Richtung der Regierung, die von ihrem Kurs nicht abzurücken brauchte. Im Hinblick auf die Flüchtlingskrise schränkte die Politisierung des Themas in Deutschland das Handeln der Regierung ein. Darüber hinaus dürfte der Europaskeptizismus mit dem Aufstieg der AfD und ihrem Durchbruch auf Bundesebene einen nachhaltigen einschränkenden Einfluss auf die deutsche Europapolitik ausüben. Die Innenpolitik ist nach wie vor entscheidend, aber die genauen Folgen ihrer Bedeutung für die Regierungspolitik hängen vom jeweiligen Thema ab.

Vor diesem Hintergrund musste die deutsche Europapolitik durch die unruhigen Gewässer der späten 2010er Jahre steuern. Der Review 2014 „Außenpolitik weiter denken“ des Auswärtigen Amtes kam zu dem Schluss, dass die Stärkung der europapolitischen Fähigkeiten sowohl des Auswärtigen Amtes als auch Deutschlands als solchem erforderlich ist. ${ }^{59}$ Angesichts der anhaltenden Turbulenzen in der EU scheinen diese Empfehlungen sinnvoll. Der Brexit hat die Aufmerksamkeit auf einige neue politische Herausforderungen gerichtet, insbesondere in der Sicherheits- und Verteidigungspolitik. Auf unterschiedliche Weise wurde mit der SSZ und dem Vertrag von Aachen vom Januar 2019 auf diese Umstände reagiert. Dennoch bleiben dies bescheidene Antworten. Insbesondere der Vertrag von Aachen enttäuscht, da er vor allem bezüglich der Governance der Eurozone schweigt.

Trotz allem bleibt Deutschland die unverzichtbare Gestaltungsmacht der EU. Ist die Große Koalition stabil genug, um eine proaktive Rolle bei der Bewältigung der heutigen Herausforderungen in der EU zu spielen? Können die Parteien in der politischen Mitte dazu beitragen? Verfügen die politischen Persönlichkeiten an der Spitze der deutsch-französischen Partnerschaft über ausreichend innenpolitische Autorität, damit diese einen Weg nach vorne weisen kann? Das sind Fragen, auf die wir die Antworten noch nicht kennen. Diese sind jedoch nicht nur für die Zukunft der Rolle Deutschlands, sondern auch für die Zukunft der EU selbst von zentraler Bedeutung.

Übersetzung aus dem Englischen: Yvonne Braun und Sabine Hoscislawski.

59 Annegret Bendiek: Abschied von der Juniorpartnerschaft. Für mehr deutsche Führung in und durch Europa, in: Internationale Politik 5/2014, S. 60ff.; Josef Janning: Germany's Foreign Ministry reinvents itself, in: European Council on Foreign Relations, 18. März 2015. 